\title{
XPS AS CHARACTERIZATION TOOL FOR PV: FROM THE SUBSTRATE TO COMPLETE III-V MULTIJUNCTION SOLAR CELLS
}

\author{
M. Gabás ${ }^{1}$, M.C. López-Escalante ${ }^{1}$, C. Algora ${ }^{2}$, I. Rey-Stolle ${ }^{2}$, E. Barrigón ${ }^{2}$, I. García ${ }^{2}$, B. Galiana ${ }^{2,3}$, S. Palanco ${ }^{1}$, S. \\ Bijani $^{1}$ and J.R. Ramos-Barrado ${ }^{1}$. \\ ${ }_{2}^{1}$ Dpto. de Física Aplicada I, Lab. de Materiales y Superficies, Universidad de Málaga, 29071 Málaga, Spain. \\ ${ }^{2}$ Instituto de Energía Solar-Universidad Politécnica de Madrid, Avda. Complutense 30, 28040 Madrid, Spain. \\ ${ }^{3}$ Present address: Instituto de Ciencia de Materiales de Madrid, CSIC, Sor Juana Inés de la Cruz 3, 28049-Madrid, Spain
}

\begin{abstract}
This contribution aims to illustrate the potential of the Xray photoelectron spectroscopy (XPS) technique as a tool to analyze different parts of a solar cell (surface state, heterointerfaces, profile composition of ohmic contacts, etc). Here, the analysis is specifically applied to III-V multijunction solar cells used in concentrator systems. The information provided from such XPS analysis has helped to understand the physico-chemical nature of these surfaces and interfaces, and thus has guided the technological process in order to improve the solar cell performance.
\end{abstract}

\section{INTRODUCTION}

XPS is a widely extended surface analysis technique; it yields information on the first few nanometers of the film. Their abilities range from the identification of any contaminant or residues at the sample surface to the analysis of the orientation of adsorbed molecules or clusters into a particular surface. Photoelectron Spectroscopy (PES) of core levels is a sensitive probe of the local chemical environment around the emitting atom. When used in combination with $\mathrm{Ar}^{+}$sputtering, it can constitute a first approximation to the interface chemistry between two layers, although the effects on the sample surface due to the $\mathrm{Ar}^{+}$sputtering process (preferred sputtering, metal reduction and Ar implantation) have to be taken into account. Angle resolved XPS (ARXPS) of both, core levels and Auger transitions among them offers valuable information on the evolution of the oxidation state of a particular cation.

The interface between two layers is a critical part in any layered electronic device, since existing defects and impurities can profoundly affect the final device performance. XPS analysis can detect the formation of spurious compounds that may intensely affect the charge carrier displacement through the interface [1]. Thus, a careful look on the surfaces and interfaces appears as a first unavoidable step to achieve a complete characterization of the whole device. This analysis is especially important in the case of III-V multijunction solar cells which are made up at least by 30 different layers, of more than 6 different semiconductors, with thicknesses varying from $20 \mathrm{~nm}$ to 2 microns and with doping levels going from $10^{17}$ to $10^{20} \mathrm{~cm}^{-3}$ (see figure 1). Every surface and interface in this layered structure is a potential object of analysis using XPS techniques. Besides, a key task in these cells is the optimization of the heteroepitaxial growth of III-V layers on the germanium substrate. It requires that III-V epilayers on Ge provide a defect-free template for the subsequent epitaxial growth, which in fact is very dependent on the surface state of the Ge substrates.

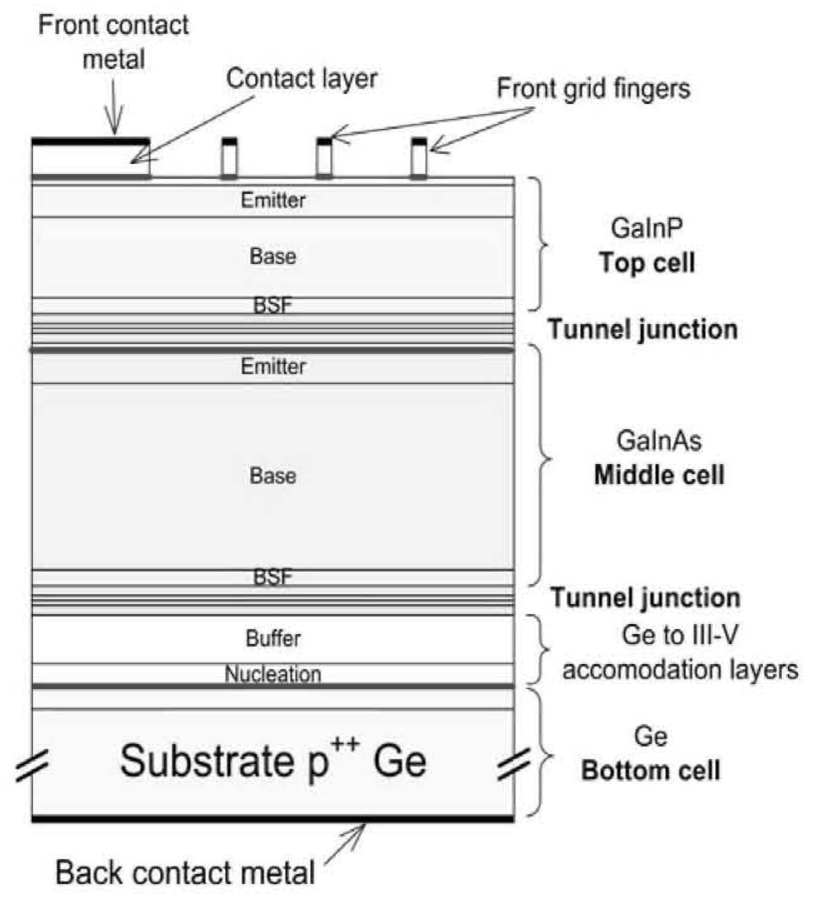

Figure 1: Schematic view of a III-V multijunction solar cell structure. Every layer is a different III-V semiconductor compound doped with the appropriate element. Red lines represent the three structure levels we have dealt with in this work.

Accordingly, the purpose of this paper is to show the potential of these techniques when applied to solar cell characterization and to use them in solving problems related with III-V multijunction solar cells. Three specific problems at different cell structure levels have been chosen to exemplify the capabilities of the technique: 1) the presence of residuals and the $\mathrm{Ge}$ chemical state in the $\mathrm{Ge}$ substrate, 2) the chemistry at intermediate layer surfaces and interfaces, and 3 ) the formation of spurious phases on the ohmic contacts. 


\section{ANALYSIS AT THE SUBSTRATE LEVEL}

A good epitaxy starts with a thorough understanding of the substrate surface. The presence of oxides, contaminants or adsorbates can have a key role in the formation of defects during the subsequent III-V layer growth [2]. Now we present the study carried out on the surface state of commercial epi-ready $\mathrm{Ge}$ wafers from two different vendors, namely wafer $A$ and $B$. Irrespective of the vendor, XPS analysis shows that all the Ge wafers have an oxide layer and reveals the presence of carbon and, probably chlorine, although the last element seems to appear in a very little quantity. In wafers $A$, carbon is present in the form of carbonates and there are some indications that traces of potassium, through the K2p core level signal, could appear close to the C1s signal, but the confirmation of its presence needs further experimental work. There are no traces of other contaminants, such as fluorine, sodium or nitrogen in any of the wafers.

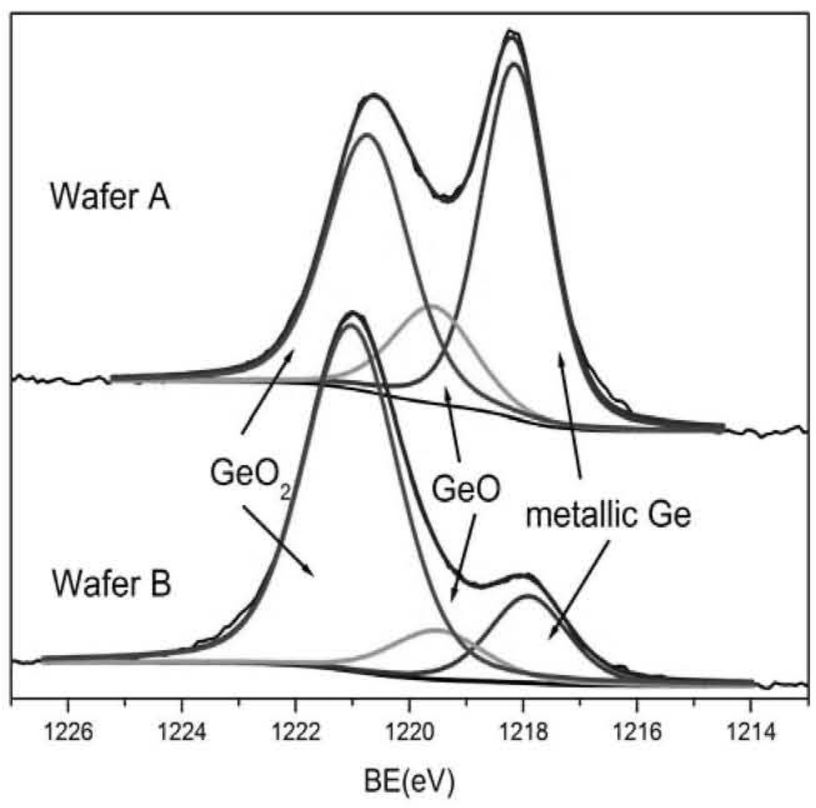

Figure 2: Ge2p spectra of two different Ge wafers, showing the three contributions: metallic $\mathrm{Ge}, \mathrm{GeO}$ and $\mathrm{GeO}_{2}$ in different proportions.

The very different shape of the Ge core level peaks inform us that the mean oxidation state of the Ge element is not the same in these two wafers, being wafers $B$ much more oxidized. The differences in the $\mathrm{Ge}$ mean oxidation state are presented in figure 2, where the $G e 2 p$ spectra from two different wafers, namely $A$ and $B$, are depicted. Three components constitute the core level signal in both cases: metallic $\mathrm{Ge}, \mathrm{GeO}$ and $\mathrm{GeO}_{2}$, although their relative intensities differ from one wafer to another. No other suboxides have been detected. As it is evident in this figure, the related weight of oxidized $\mathrm{Ge}$ is larger in wafers $\mathrm{B}$, which implies a thicker oxide layer on these wafer surfaces. Ge 3d core level signals (not shown) allow to perform the same analysis and to reach identical conclusions relative to the differences in the Ge oxidation state.

01s spectra also evidence the different mean oxidation state of $\mathrm{Ge}$ wafers coming from different vendors (see Fig. 3). Two contributions coming from $\mathrm{GeO}$ and $\mathrm{GeO}_{2}$, appear in all the wafers. Exactly as it happens for the $\mathrm{Ge}$ core level spectra, the deconvolution of the total 01s XPS signal among their components yields a ratio $\mathrm{GeO}_{2} / \mathrm{GeO}$ bigger for the wafers $\mathrm{B}$. Moreover, in the wafers where the presence of carbonates has been detected in the $\mathrm{C} 1 \mathrm{~s}$ spectrum -wafers coming from vendor A-, there is an extra contribution to the $01 \mathrm{~s}$ core level signal in the high binding energy region (see Fig. 3 top), which does not appear in any of the O1s and C1s core level spectra from wafers B. There is no other contribution from any other $\mathrm{C}-\mathrm{O}$ compound in none of the wafers.

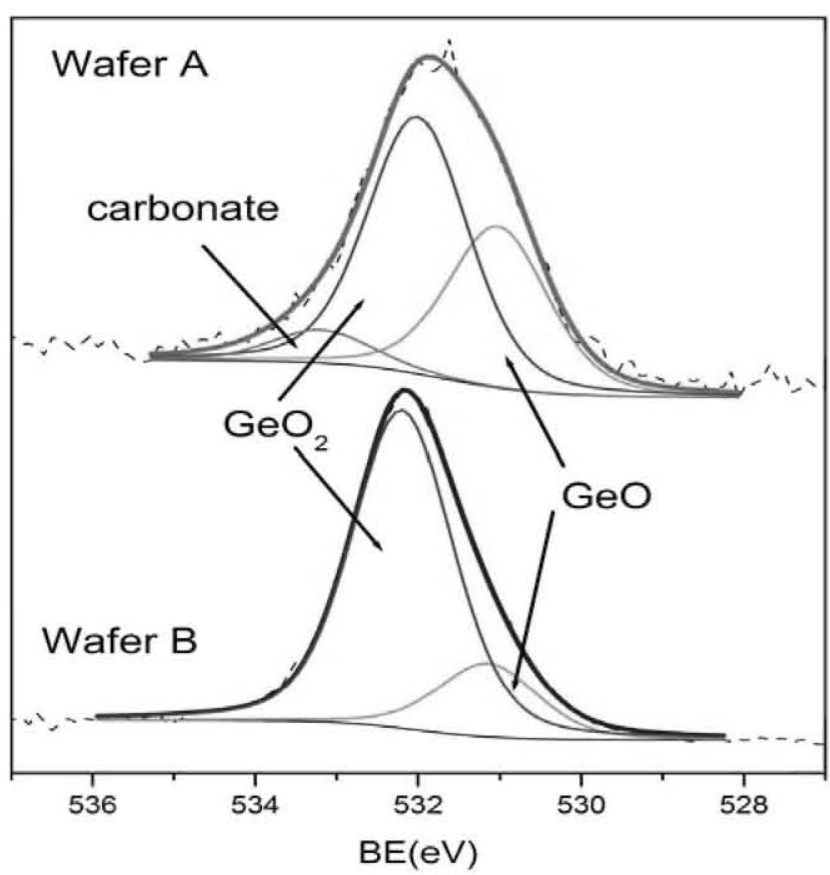

Figure 3: 01s spectra of the same two wafers as in figure 2, showing their contributions with different relative intensities.

Our results state the differences in the surface chemical state of wafers coming from different vendors, with an oxide layer thickness strongly dependent on the wafer origin. The global analysis of the XPS data indicates that the presence of carbonates seems to inhibit the oxide layer growth. It is foreseen that oxide layers can be easily removed during the epitaxial process; whilst the removal of carbonates might be a challenging issue. Therefore, XPS analysis of the Ge wafer surface supplies very useful information for the subsequent epitaxial growth of III-V layers. The core level analysis allowed to distinguish the different surface chemical state of $\mathrm{Ge}$ in wafers supplied by two vendors and to identify the surface contaminants. 


\section{ANALYSIS OF THE SEMICONDUCTOR STRUCTURE}

As an example of the various applications that XPS techniques allow to tackle, we present in this section the study of the oxidation of $\mathrm{GaAs}$ and GalnP layers when they are grown onto GalnP and GaAs templates respectively, by Metal Organic Vapor Phase Epitaxy (MOVPE). ARXPS has been used for this purpose. The basis of this technique is that photoelectrons getting out from the sample with different take-off angles respect to the surface layer, provide information from different depths in the film, taking into account that the photoelectrons mean free path is only dependent on both matrix effects and photoelectron kinetic energy. Thus, data taken at small take-off angles supply information of the very first layers.

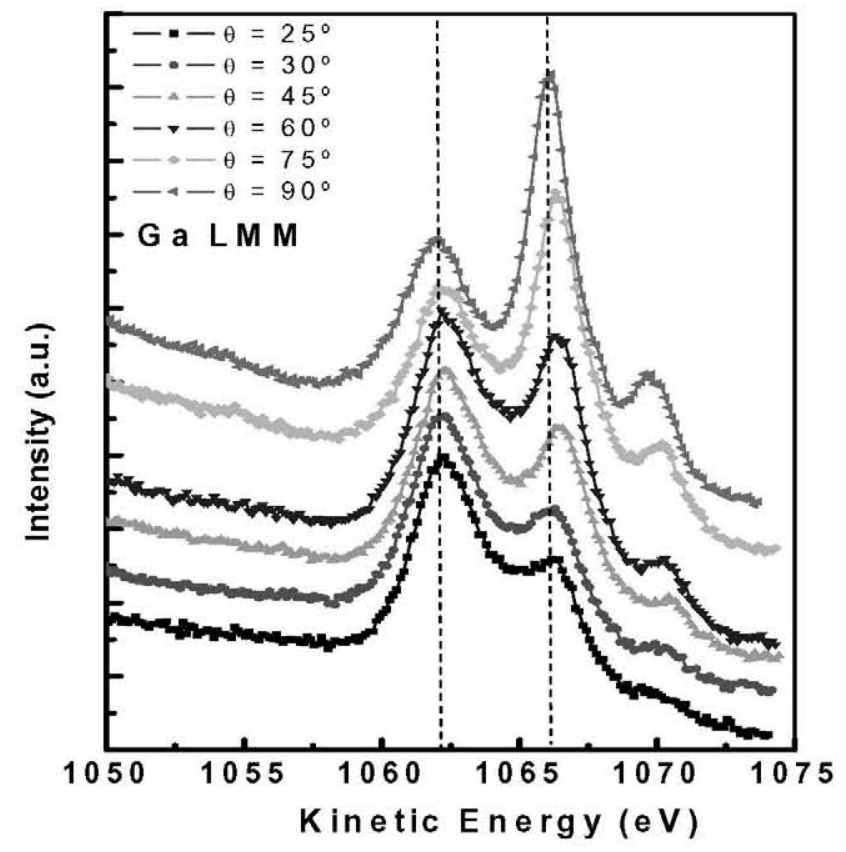

Figure 4: Ga LMM spectra at different take-off angles, probing the $\mathrm{Ga}$ oxidation state at different depths in the GaAs layer. Small angles correspond to more superficial layers.

The surface $\mathrm{Ga}$ oxidation state in a $\mathrm{GaAs}$ layer deposited onto a GalnP template has been examinated through the evolution of the Ga LMM Auger spectrum with probing depth. Figure 4 shows these depth-dependent spectra, with a dominant peak centered at $\sim 1062 \mathrm{eV}$ for small takeoff angles while for take-off angles close to $90^{\circ}$, the dominant peak is centered at $\sim 1066 \mathrm{eV}$. The corrected Auger parameter corresponding to these energies are $1082.74 \mathrm{eV}\left(\mathrm{Ga}_{2} \mathrm{O}_{3}\right)$ and $1085.6 \mathrm{eV}$ (GaAs). Therefore, from the data shown in figure 4 , it can be deduced that the GaAs surface presents a superficial oxide layer, but its thickness is small, (less than $2 \mathrm{~nm}$ ). On the other hand, the corrected Auger parameter corresponding to As maintains a rather constant value between $1266.2 \mathrm{eV}$ and $1266.5 \mathrm{eV}$, which are typical values of GaAs compound, indicating that the As oxidation is almost negligible in the GaAs layer.

On the contrary, when the GalnP layer is grown onto a GaAs layer, the Ga LMM Auger spectra shows that the presence of $\mathrm{Ga}$ oxide is almost negligible at the surface of the layer as it has been previously reported by the authors [3]. Therefore, $\mathrm{Ga}$ cation is oxidized more easily when exposed to air in the GaAs lattice than in the GalnP compound. Arsenic core level signals are also detected at the GalnP surface, showing up the possible As segregation from the interface trough the GalnP layer.

Another fundamental problem in III-V solar cells is the cross-diffusion of different atoms at the various interfaces in the structure. The interfaces between two III-V semiconductors have received so much attention because of their fundamental role on the performance of the solar cell device. In that way, the in-depth resolution that XPS can supply is of great relevance in order to determine the abruptness of a heterojunction. Accordingly, a first approximation to the interface $\mathrm{GaAs} / \mathrm{GalnP}$ has been made by using XPS combined with $\mathrm{Ar}^{+}$sputter depth profiling. However, several unintended effects could accompany this technique and must be taken into account.

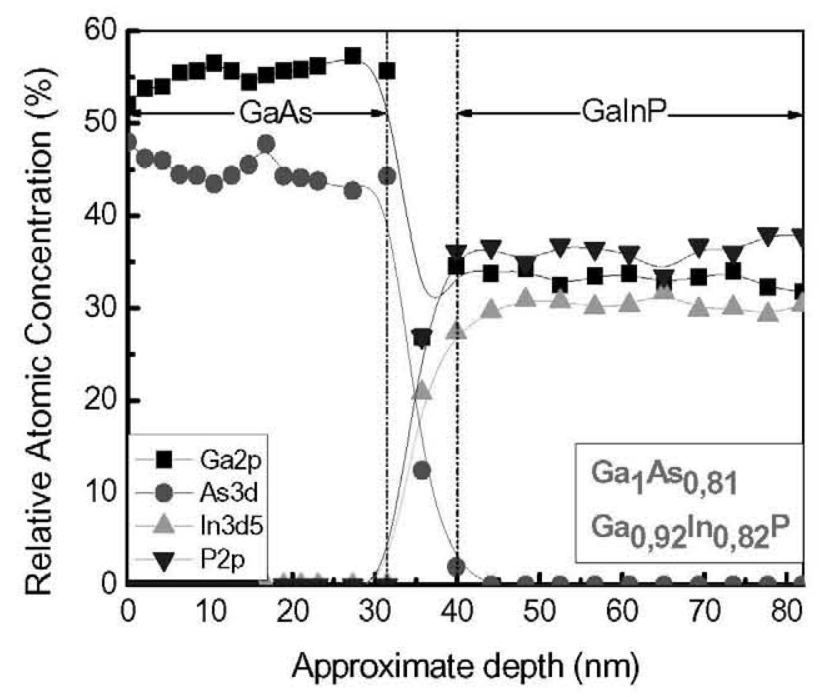

Figure 5: XPS depth profile of the GaAs/GalnP heterointerface.

On the left part of the graph presented in figure 5 , the $\mathrm{Ga}$ and As signals corresponding to the $\mathrm{GaAs}$ layer appear as rather flat and constant contributions, but the relative atomic concentrations obtained (see inset), differ from the real layer stoichiometry. The origin of the As deficiency may be attributed to preferential sputtering of As from the GaAs matrix, due to the fact that the As sublimation energy has a value of $130.12 \mathrm{~kJ} \cdot \mathrm{mol}^{-1}$, which is smaller 
than that of the $\mathrm{Ga}\left(271.72 \mathrm{~kJ} \cdot \mathrm{mol}^{-1}\right)$. As it is shown in figure 5 , the interface is characterized by a strong decay in the $\mathrm{Ga}$ and As atomic concentrations, which is indicative of a rather abrupt interface. This decrease of $\mathrm{Ga}$ and As signals is accompanied by the appearance of the In and $P$ core level signals. The great diferrence in stoichiometry also measured for the GalnP layer (expected to be $\left.\mathrm{Ga}_{0.5} \mathrm{In}_{0.5} \mathrm{P}\right)$ can be also explained analogously in terms of preferential sputtering of the some of the species in the alloy.

\section{ANALYSIS AT THE DEVICE LEVEL}

A great deal of attention and effort has been directed towards the achievement of high quality ohmic contacts to $\mathrm{GaAs}$ films and other III-V semiconductors in order to diminish the solar cell series resistance. In the case of concentrator solar cells, these requirements are especially important because the ohmic contact must exhibit simultaneously a low metal sheet resistance and a low specific contact resistance $[4,5]$. Cu/Ge alloys could be a good alternative due to their electrical characteristics and low cost as compared to conventional gold or silver-based alloys.

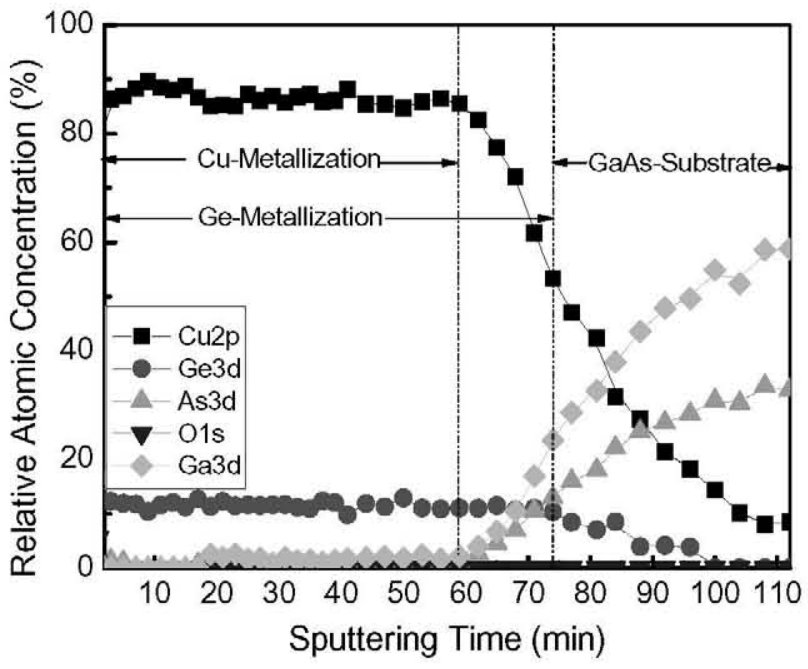

Figure 6: XPS depth profile of the layer $\mathrm{Cu} / \mathrm{Ge}$ and its interface with the GaAs layer.

In order to study the interface between $\mathrm{Cu} / \mathrm{Ge}$ and $\mathrm{GaAs}$ layers, XPS measurements combined with $\mathrm{Ar}^{+}$sputtering have been conducted. Epitaxially grown n-GaAs layers were coated with a $\mathrm{Cu} / \mathrm{Ge}$ alloy $(800 \mathrm{~nm}$ thick) prepared by sequential thermal evaporation of a Cu layer (500nm thick) followed by a $\mathrm{Ge}$ layer (300nm thick). A subsequent annealing process at high temperature formed the ohmic contact as well as the $\mathrm{Cu} / \mathrm{Ge}$ alloy. Before any cleaning procedure, XPS results show that at the topmost of the surface only $\mathrm{Cu}, \mathrm{O}$ and $\mathrm{C}$ signals are detected. $\mathrm{Cu}$ is oxidized at the surface of the layer, but just 1 minute of sputtering with $\mathrm{Ar}^{+}$ions is enough to make both, the copper oxides and carbon disappear [6]. After the superficial contamination has been eliminated, only $\mathrm{Cu}$ and $\mathrm{Ge}$ appear in XPS spectrum, showing a metallic layer without any trace of $\mathrm{Ge}$ oxides. A continuous $\mathrm{Ar}^{+}$ sputtering process accompanied by XPS analysis of the $\mathrm{Cu} 2 \mathrm{p}, \mathrm{Ge} 3 \mathrm{~d}, \mathrm{O} 1 \mathrm{~s}, \mathrm{As} 3 \mathrm{p}$ and Ga3d signals allows to confirm the formation of a metallic $\mathrm{Cu} / \mathrm{Ge}$ alloy with a homogeneous composition of $\mathrm{Cu}$ and $\mathrm{Ge}$, as it is evident from the flat signal intensity vs. time signal for both elements (see Fig. 6).

When the interface with the GaAs layer is reached, the Ge3d core level maintains a constant signal up to a greater depth than $\mathrm{Cu} 2 \mathrm{p}$ does. In this region, $\mathrm{As}$ and $\mathrm{Ga}$ core levels can also be detected. It seems that a mixture based on $\mathrm{Ge}$ and $\mathrm{Cu}$, together with $\mathrm{Ga}$ and $\mathrm{As}$ has been formed. This layer could be the responsible for the low specific contact resistance reported and thus, for the good performance of the $\mathrm{Cu} / \mathrm{Ge}$ alloy as ohmic contact [7]. There is no indication of the formation of phases like $\mathrm{GeAs}_{2}$ at the interface. The evolution of the Ge3d, Cu2p core level binding energies and corrected Auger parameters for both elements (see fig. 7) are only compatible with metallic $\mathrm{Ge}$ and $\mathrm{Cu}$. This information has been crucial for us in order to optimize this kind of ohmic contact.

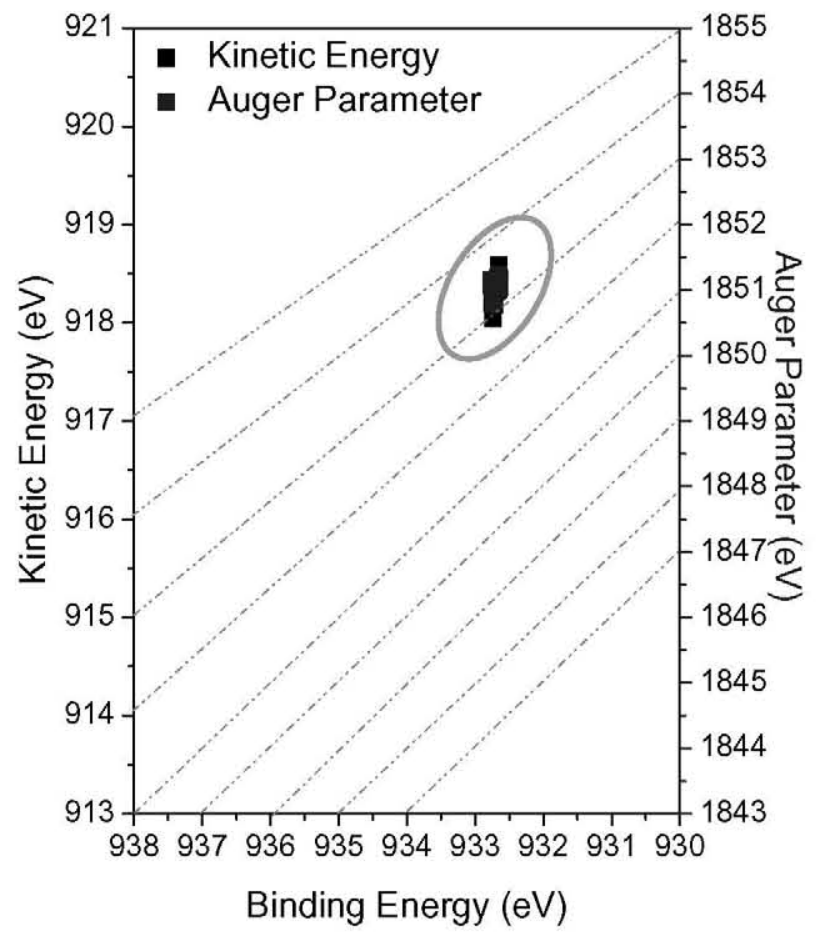

Figure 7: Chemical state plot for corrected Auger parameter of the Cu LMN transition.

\section{SUMMARY AND CONCLUSSIONS}

XPS has proved to be a powerful technique for the characterizaction of solar cell materials at diverse stages 
of the fabrication process. Several techniques based on XPS have been used to solve different problems related to the various layers, in the III-V multijunction solar cell structure. The detection of surface contaminants, the identification of the chemical state of the elements in a surface, the study of the length and composition of interfaces and the formation of intermediate compounds in the ohmic contacts, are some of the problems where XPS sheds light and offers a valuable knowledge inaccessible when using some other techniques.

\section{ACKNOWLEGDMENTS}

This work has been supported by the Spanish Ministerio de Educación y Ciencia with the CONSOLIDER-INGENIO 2010 program by means of the GENESIS FV project (CSD2006-004). The Spanish Ministerio de Ciencia e Innovación has also contributed with the SIGMASOLES project (PSS-440000-2009-30) and with the projects with references TEC2008-01226, TEC2009-11143 and TEC2010-16700, as well as the Comunidad de Madrid under the NUMANCIA II programme (S2009/ENE1477) and project CCG10-UPM/ENE-566.

\section{REFERENCES}

[1] M. Gabás, N.T. Barrett, J.R. Ramos-Barrado, S. Gota, T.C. Rojas, M.C. López-Escalante, "Chemical and electronic interface structure of spray pyrolysis deposited undoped and Al-doped $\mathrm{ZnO}$ thin films on a commercial Cz-Si solar cell substrate", Solar Ener. Mat. \& Solar Cells, 93, 2009 pp. 1356-1365.

[2] I. Rey-Stolle E. Barrigón, B. Galiana, C. Algora, "Analysis of germanium epiready wafers for III-V heteroepitaxy", J. Crystal Growth, 310, 2008 pp. 48034807.

[3] M.C. López, B. Galiana, C. Algora, I. Rey-Stolle, M. Gabás, F. Martín, J.R. Ramos-Barrado "Chemical characterization of $\mathrm{GaAs} / \mathrm{lnGaP}$ heterointerfaces grown by metal organic vapor phase epitaxy (MOVPE) by XPS and ARXPS", 22nd Eur. Photovolt. Solar Energy Conf., 2007, pp. 253-255

[4] C. Algora, V. Díaz, "Influence of series resistance on the guidelines for the manufacture of concentrator $\mathrm{p}$-on-n GaAs solar cells", Prog. Photovolt: Res. Appl. 8, 2000 pp. 211-225.

[5] B. Galiana, C. Algora, I. Rey-Stolle "Comparison of 1D and $3 \mathrm{D}$ analysis of the front contact influence on GaAs concentrator solar cell performance", Solar Ener. Mater. \& Solar Cel/s 90, 2006, pp. 2589-2604

[6] M.C. López, B. Galiana, C. Algora, I. Rey-Stolle, M. Gabás, J.R. Ramos-Barrado, "Chemical characterization by XPS of $\mathrm{Cu} / \mathrm{Ge}$ ohmic contacts to n-GaAs", Appl. Surf. Sci. 253, 2007 pp. 5062-5066.
[7] I. Rey-Stolle, B. Galiana and C. Algora, "Assessment of a low-cost gold-free metallization for III-V high concentrator solar cells", Solar Ener. Mater. \& Solar Cells 91, 2007, pp. 847-850. 